\title{
Locally reinforced polymer-based composites for efficient heat dissipation of local heat source
}

\author{
Chao Yuan, Lan Li, Bin Duan, Bin Xie, Yongming Zhu, \& Xiaobing Luo* \\ State Key Laboratory of Coal Combustion, School of Energy and Power Engineering, Huazhong \\ University of Science and Technology, Wuhan, 430074, China \\ *Corresponding author: luoxb@ hust.edu.cn
}

\begin{abstract}
Local heat source in electronic device is likely to produce hot spot which can degrade the reliability and performance of the device. Various materials have been attempted to enhance the heat dissipation of local heat source. Many theoretical studies have demonstrated that the heterogeneous composite materials with fillers concentrated at the preferential paths of heat flux are effective in cooling the local heat source. However, this unique control of microstructure and property for polymer-based composites has less been achieved in practice due to the technical difficulties in controlling the fillers positions. In this paper, a locally reinforced heterogeneous composite with conductive particles concentrated at the preferential path of heat flux was fabricated to cool the local heat source. The local reinforcement was achieved by using magnetically responsive particles as reinforcing elements and a specific magnetic field to organize the elements into the predefined structure. To evaluate the thermal performance of the proposed material, we performed the comparative thermal tests. The results show that compared to the homogeneous composites, the present composites with local reinforcement can significantly enhance the heat dissipation of local heat source. When heat flux is $5840 \mathrm{~W} \mathrm{~m}^{-2}$, the locally reinforced composites with a fillers volume fraction of $5 \%$ reduced the average and maximum temperature of heater $7.7{ }^{\circ} \mathrm{C}$ and $8.7{ }^{\circ} \mathrm{C}$, respectively.
\end{abstract}

Keywords: polymer-based composites; heat dissipation; local heat source; hexagonal boron nitride; magnetic control

\section{Nomenclature}

D diameter of platelet, $\mu \mathrm{m}$ 


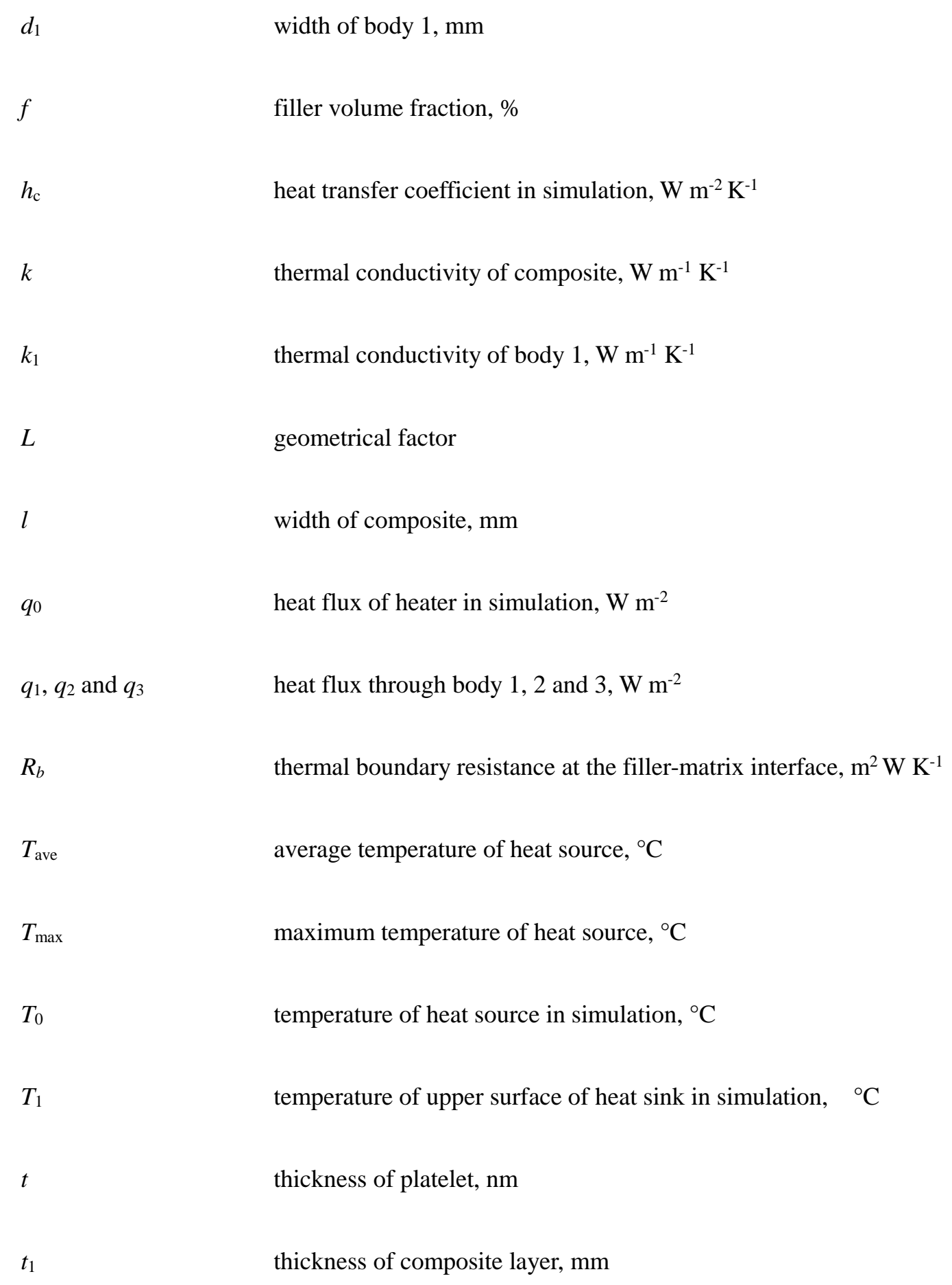

heat transfer coefficient in simulation, $\mathrm{W} \mathrm{m}^{-2} \mathrm{~K}^{-1}$

thermal conductivity of composite, $\mathrm{W} \mathrm{m}^{-1} \mathrm{~K}^{-1}$

thermal conductivity of body $1, \mathrm{~W} \mathrm{~m}^{-1} \mathrm{~K}^{-1}$

geometrical factor

width of composite, $\mathrm{mm}$

heat flux of heater in simulation, $\mathrm{W} \mathrm{m}^{-2}$

heat flux through body 1,2 and $3, \mathrm{~W} \mathrm{~m}^{-2}$

thermal boundary resistance at the filler-matrix interface, $\mathrm{m}^{2} \mathrm{~W} \mathrm{~K}^{-1}$

average temperature of heat source, ${ }^{\circ} \mathrm{C}$

maximum temperature of heat source, ${ }^{\circ} \mathrm{C}$

temperature of heat source in simulation, ${ }^{\circ} \mathrm{C}$

temperature of upper surface of heat sink in simulation, $\quad{ }^{\circ} \mathrm{C}$

thickness of platelet, $\mathrm{nm}$

thickness of composite layer, $\mathrm{mm}$

\section{Greek symbols}

$\alpha$

inverse of filler aspect ratio 


$\begin{array}{ll}\Delta T & \begin{array}{l}\text { temperature difference betw } \\ \text { sink, }{ }^{\circ} \mathrm{C}\end{array} \\ \Delta T_{\text {ave }} & \text { reduction of average tem } \\ \Delta T_{\max } & \text { reduction of maximum te } \\ \text { Subscripts } & \\ 11 & \text { in-plane direction } \\ 33 & \text { through-plane direction } \\ m & \text { matrix } \\ p & \text { particles }\end{array}$

\section{Introduction}

Over the past few decades, the revolution in electronics has resulted in the packaging of multiple functional units on the same chip $[1,2]$. These units create a non-uniform distribution of heat source throughout the chip. The local heat source with high power is likely to produce hot spots. These hot spots can lead to excessive stresses on the chip, which degrade the reliability and performance of electronic devices [1-4]. Thermal management of local heat source is a big challenge. Various materials [5-7] have been attempted to enhance the heat dissipation. Thermally conductive polymer-based composites, fabricated by incorporation of highly conductive fillers, are potential for the thermal management materials [8-11]. However, applying the conventional composites that possess uniform thermal properties for the local heat source cooling is still insufficient and not cost-effective [12].

Many theoretical studies [13-17] have demonstrated that the heterogeneous composite materials with fillers concentrated at the preferential paths of heat flux are effective in cooling the local heat source. For instance, some topology designs [13-15] have been applied to the composite material which is loaded with local heat source, to minimize the heat source temperature. These designs are accomplished by locating the fillers in matrix with optimal distribution using the optimization algorithm. The designed results show that concentrating the fillers at the position with large temperature gradient is most efficient to decrease the heat source temperature. In addition, the percolation theory $[16,17]$ can further illustrate the benefit of fillers local concentration. Percolation 
happens when the high conductivity particles form at least one continuous chain in composites from heat source to sink. At that time, there is a sudden increase in thermal performance [17]. Fig. 1(a) schematically shows a homogenous composite with low fillers loading assembled with local heat sources. Percolation hardly takes place in this composite since all particles are well separated. While, as seen in Fig. 1(b), concentrating the fillers makes it more probable to form the continuous chains resulting in a local reinforcement at the region with local heat source.

a

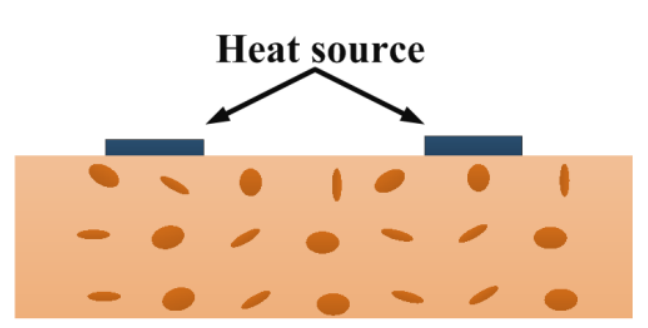

Fig. 1. Schematic of (a) homogenous composite an reinforcement. b

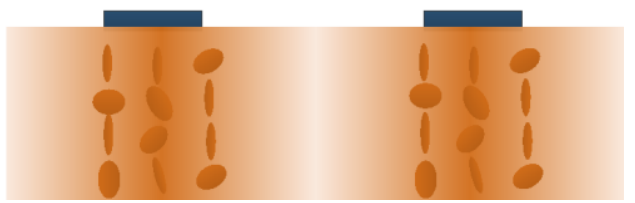

(b) heterogeneous composite with local

The theoretical studies above well illustrate the benefit of fillers local concentration. However, this unique control of microstructure and property for polymer-based composites has less been achieved in practice due to the technical difficulties in controlling the fillers positions. Squeezing [18-20] is a process which can make fillers entrapped and compacted in matrix. The heterogeneity of composites occurs when the rate of matrix flow through the fillers is greater than that of composites deformation $[19,20]$. However, this method is limited to the thin films and demands highly accurate control of the squeeze rate $[19,20]$. Electric field [21] is another approach to achieve the unique microstructure, in which the localization of fillers is induced by electric field concentration. But this approach requires ultrahigh DC electric field $(1 \mathrm{kV})[21]$ and multiple processing steps. Recently, an attractive strategy is proposed to control the distribution of fillers in matrix [22, 23]. The approach relies on coating the non-magnetic reinforcing particles with superparamagnetic nanoparticles. These coated particles exhibit an ultrahigh magnetic response (UHMR) [23] which enables remote control over their distribution under low external magnetic fields in low-viscosity suspending fluids. Such fluids can be then consolidated to fix the magnetically-imposed distribution and thus produce the composites with deliberately tuned properties. With this strategy, the former investigations [23, 24] have shown the possibility in fabricating the polymeric substrate with locally tuned mechanical properties. A careful review of literature indicates that this strategy has not been applied for tailoring the composites thermal properties. In addition, although the reports $[20,21]$ have fabricated the composite with deliberately controlled microstructure, experiments have not been conducted to evaluate its thermal performance.

In this research, we report that the polymer-based composites applied for heat dissipation from 
local heat source can be reinforced by concentrating the thermally conducting fillers at the preferential paths of heat flux. The locally reinforced composite is fabricated by using the magnetically responsive thermally conducting particles as reinforcing elements and a specific magnetic field to control the elements distribution. Then, a thermal testing system is built to evaluate the composites thermal performance. Finally, we conduct a simulation on the constructed composite to thoroughly investigate its thermal property.

\section{Experiments}

\subsection{Synthesis of locally reinforced polymer-based composites}

Polymer-based composite is composed of polymer matrix and reinforcing particles. In this report, we employed the silicone gel (OE-6550, Dow Corning), with a viscocity of $4 \mathrm{~Pa}$ s and a relatively low thermal conductivity $\left(0.16 \mathrm{~W} \mathrm{~m}^{-1} \mathrm{~K}^{-1}\right)$ [25], as the soft matrix. Hexagonal boron nitride (hBN) platelets (AC-6041, Momentive), with an average diamater of $5 \mu \mathrm{m}$ and a much high in-plane thermal conductivity $\left(600 \mathrm{~W} \mathrm{~m}^{-1} \mathrm{~K}^{-1}\right)$ [26-27], were chosen as the reinforcing elements. To gain magnetic control of the reinforcements, the platelets were coated with $2 \mathrm{wt} \%$ superparamagnetic iron oxide nanoparticles via a previously reported procedure [26-27]. hBN platelets $(4 \mathrm{~g})$ were first stirred in deionized water $(200 \mathrm{ml})$ at $\mathrm{pH}=7$. Then, EMG-605 ferrofluid (Ferrotec, U.S.A) $(400 \mu \mathrm{L})$ diluted with

deionized water $(5 \mathrm{ml})$ was added dropwise to the suspension under vigorous stirring. The $\mathrm{pH}$ of suspension was held at 7 to keep a negative charge on the surface of hBN platelets. The EMG-605 ferrofluid is an aqueous suspension containing iron oxide nanoparticles coated with a cationic surfactant, which enables electrostatic adsorption of the positively charged magnetic nanoparticles onto the platelets surface. The suspension was incubated for 1 hour to allow the bonding between the platelets and iron oxide nanoparticles. After that, the coated platelets were filtered and dried for 12 hours at $90^{\circ} \mathrm{C}$ in vacuum.

Reinforced composites were produced by adding the magnetically responsive platelets to the fluidic silicone gel followed by mold casting and curing through polymerization. Magnetically responsive platelets in powder form were first added to the silicone gel and stirred for $30 \mathrm{~min}$ to be fully dispersed. Bubbles introduced during the stirring process were removed by applying alternating cycles of vacuum. The resulting suspension was then poured into a $26 \mathrm{~mm} \times 26 \mathrm{~mm} \times 2 \mathrm{~mm}$ (length $\times$ width $\times$ height) Teflon mold. During the mold casting, a magnetic field should be applied on the cast sample to concentrate the platelets to the specific region of interest. Fig. 2(a) gives the diagram which schematically shows the approach of locally concentrating the platelets. Two $45 \mathrm{~mm} \times 3 \mathrm{~mm} \times 3 \mathrm{~mm}$ rare earth magnets are placed symmetrically below and above the sample. The distance between the magnet and sample was $6 \mathrm{~mm}$. These magnets can create a magnetic field gradient on the sample: the strength of magnetic field between the magnets is largest and decreases gradually towards the sides of sample. The largest strength of magnetic field is $26 \mathrm{mT}$ meassured by a Gaussmeter. According to Ref. 
[24], the magnetically responsive platelets can be attracted to the place with maximum magnetic field. In this work, after 1 hour incubation, the magnetically responsive platelets were highly concentrated between the magnets. Samples with a field were heated at $60^{\circ} \mathrm{C}$ for pre-cure. An annealing step at $150^{\circ} \mathrm{C}$ for 6 hours was conducted to ensure the good adhesion between the hBN platelets and silicone matrix.

a

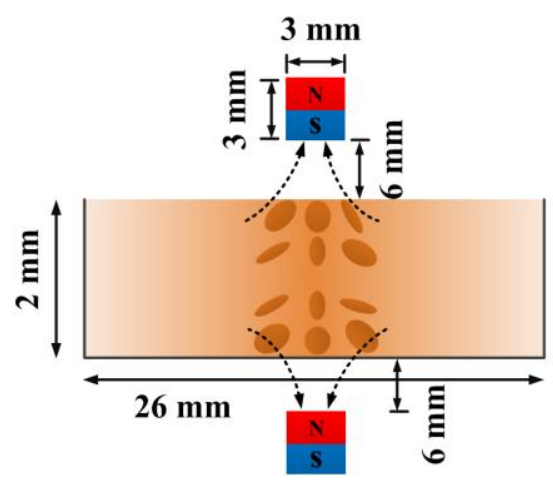

b

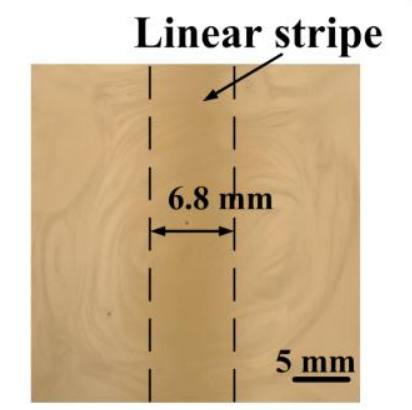

c

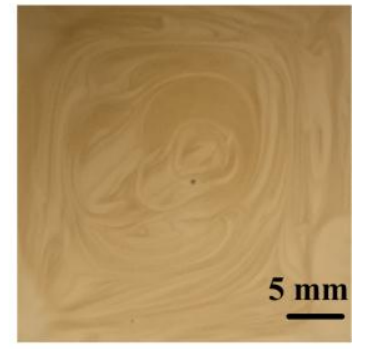

Fig. 2. (a) Schematic diagram showing the approach of locally concentrating the platelets; Photographs of prepared (b) locally structured and (c) homogeneous composites.

Fig. 2(b) shows a fabricated locally reinforced composite with a filler volume fraction of 5\%. A $6.8 \mathrm{~mm}$-wide linear stripe with a high local concentration of platelets is observed at the composite. For the comparative purpose, Fig. 2(c) presents the homogeneous composites with the same volume fraction prepared without magnetic field. According to the figure, there is no sign of platelets concentration.

\subsection{Evaluation of locally reinforced composites thermal performance}

When a specific region of composite is applied with local heat source, the accumulation of platelets in that region is expected to improve the heat dissipation and hence decrease the temperature of heat source. To demonstrate such potential, we performed comparative thermal tests on the homogeneous and locally structured samples, which were applied with the same local heat source. In the tests, the temperature response of heat source to the two samples was compared.

Fig. 3 schematically illustrates the testing system and the experimental processes. The system consists of six components: heater, samples, heat sink, supporting plates, rare earth magnets, and infrared radiation (IR) thermometer. To create local heat source, a small polyimide film heater with dimensions of $26 \mathrm{~mm} \times 8 \mathrm{~mm} \times 0.2 \mathrm{~mm}$ was attached on the samples with dimensions of $26 \mathrm{~mm} \times 26$ $\mathrm{mm} \times 2 \mathrm{~mm}$ and energized with certain power. Since it was difficult to mount the heater on the solid state composites, we employed the uncured composites as the testing samples. Good contact between the heater and samples was achieved by the adhesive force from fluidic samples. Another advantage of 
using fluidic samples was that the comparative tests could be conducted at the same base material, ensuring the same testing conditions. As seen in Fig. 3, a block (aluminium-6061 T4) with a square groove on surface was applied to hold the samples. And the lower part of block acted as the heat sink. The overall dimension of the block is $29 \mathrm{~mm} \times 29 \mathrm{~mm} \times 7 \mathrm{~mm}$, and the dimension of groove is 26 $\mathrm{mm} \times 26 \mathrm{~mm} \times 2 \mathrm{~mm}$. Two plates made of aluminium were used to support the block. The rare earth magnets were placed below and above the sample when necessary. Temperature field was measured by the IR thermometer (SC620, FLIR) fixed on top of heat source with a height $30 \mathrm{~cm}$. The temperature resolution of this instrument is $\pm 0.1^{\circ} \mathrm{C}$ and the emissivity of heat source (polyimide) was calibrated and equal to 0.95 . All the experiments were conducted at the environmental temperature of $15{ }^{\circ} \mathrm{C}$.

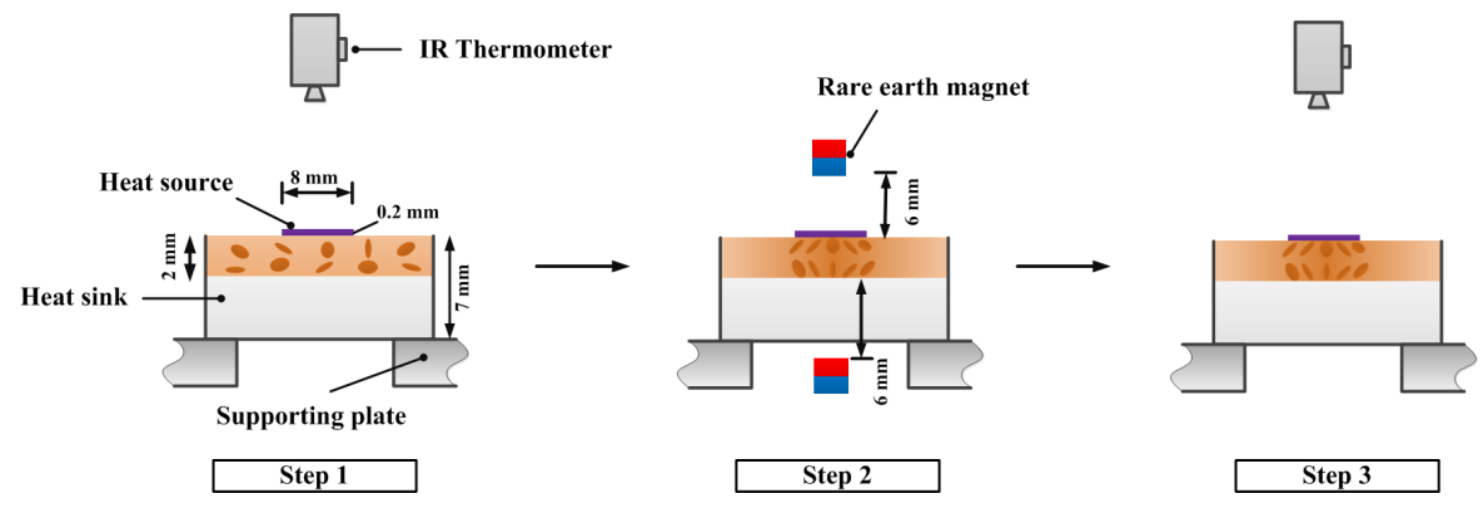

Fig. 3. Descriptions of the testing system and experimental processes.

The experiment processes can be generalized as three steps:

(1) A constant heat flux is applied on the fluidic homogeneous composites. It takes about 10 minutes to reach steady state. After that, measure the steady-state temperature field of heater.

(2) Place the rare earth magnets above and below the sample. Afer about 10 minutes, the local concentration of platelets is formed.

(3) Take away the magnets and measure the steady-state temperature field of heater with the same input power.

Generally, the silicone will cure when heated. It is possbile that thermal properties change after silicone curing and some additional heat is released to sample due to the exothermic polymerization. However, in our experiments, the silicone is hard to cure since its temperature is relative low $\left(<60^{\circ} \mathrm{C}\right)$ and the heating time is less than $30 \mathrm{~min}$. Normally, it will take 48 hours for silicone completely curing at $60{ }^{\circ} \mathrm{C}$. Therefore, the probable effect resulting from silicone curing can be neglected. Platelets settlement is a potential effect on platelet distribution and the resulting thermal performance of sample. The density of hBN platelet and silicone is 2270 and $1140 \mathrm{~kg} \mathrm{~m}^{-3}$, respectively. The platelets will settle in silicone matrix in the case of no magnetic force (at step 1). Referring to the researches [28-29], we estimate the time for the platelets settling to bottom based on density difference, viscosity of silicone 
and dimensions of platelet. The result is about 17 hours. Thus, the probable effect resulting from settlement can also be neglected since step 1 spends only $10 \mathrm{~min}$. The small settlement velocity is due to the small density difference and platelet dimensions.

\section{Results and discussion}

\subsection{Thermal performance of locally reinforced composites}

Figs. 4(a)-4(b) give the steady-state temperature fields of the testing system before and after applying the magnetic field on the $5 \mathrm{vol} \%$ composite, respectively. In this case, the heat flux given by the heater is $3200 \mathrm{~W} \mathrm{~m}^{-2}$. Regions where the contours are white and red indicate the high temperature, while the blue contours represent the low value. The rectangular area indicated in the figures is regarded as the approximate region of heat source. Average temperature of heat source $\left(T_{\text {ave }}\right)$ is then calculated. $T_{\text {ave }}$ for the homogeneous and locally reinforced samples are $36.8{ }^{\circ} \mathrm{C}$ and $34.0{ }^{\circ} \mathrm{C}$, respectively. So the reinforced sample reduces $T_{\text {ave }}$ with $2.8^{\circ} \mathrm{C}$. The lower $T_{\text {ave }}$ indicates that the local reinforcement brings in a substantial promotion for the heat conduction from heat source to sink. To further characterize the impact of local reinforcement, we study the variations of temperature on the heater at the horizontal direction. Hot spots in the temperature contours have been indicated by the blue points. Line A lies along the heater which is attached on the homogeneous sample, and Line B is along the heater with locally reinforced sample. The variations of temperature along these two lines are plotted and shown in Fig. 4(g). It can be found that the temperature varies along the two lines with a similar trend. The temperature at the left of the lines (edge of heater) is very close and low, and increases gradually until reaching a maximum temperature $\left(T_{\max }\right)$, and then decreases towards the right edge of heater. Although the temperature variations of the two lines show similar trend, $T_{\max }$ and temperature gradients across the heater are much different. $T_{\max }$ of heat source attached on the reinforced sample is $38.3{ }^{\circ} \mathrm{C}$. There is $3.8{ }^{\circ} \mathrm{C}$ reduction compared to that $\left(42.1{ }^{\circ} \mathrm{C}\right)$ of the homogeneous case. Furthermore, there is a smoother change of temperature on the heater assembled with the reinforced composites. Therefore, the locally reinforced composite shows greater ability for the heat dissipation from local heat source. 


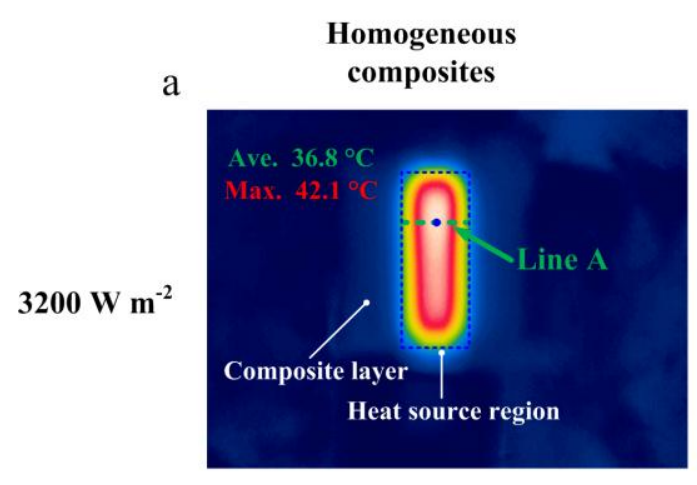

c

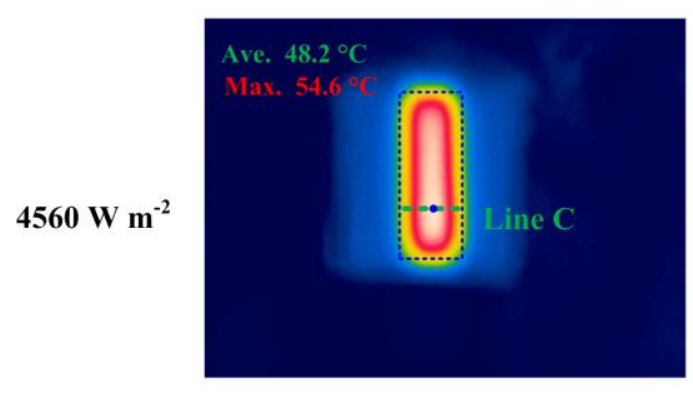

e

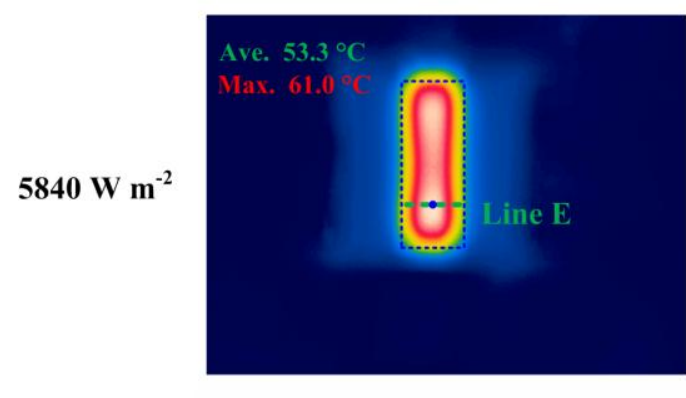

b

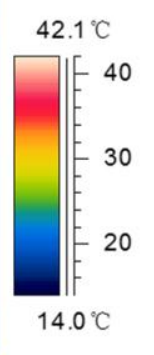

d
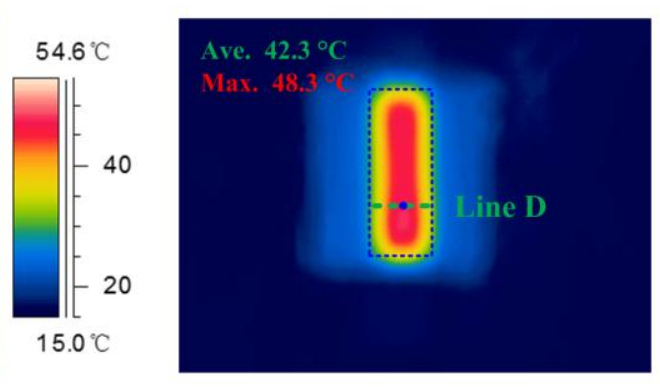

f

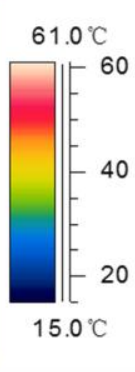

Locally reinforced

composites
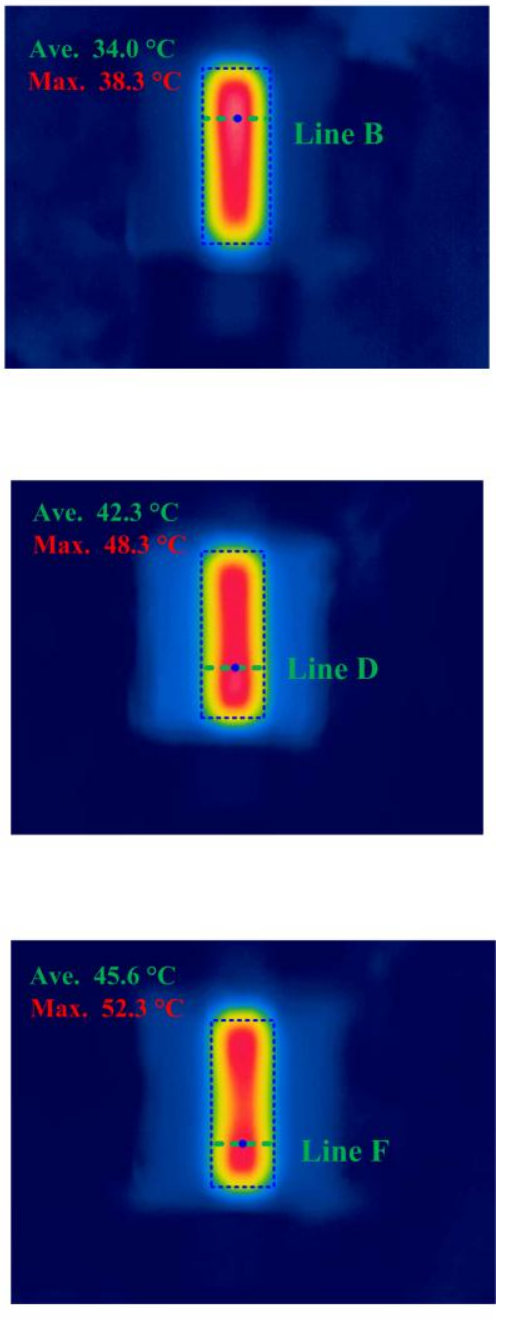

g

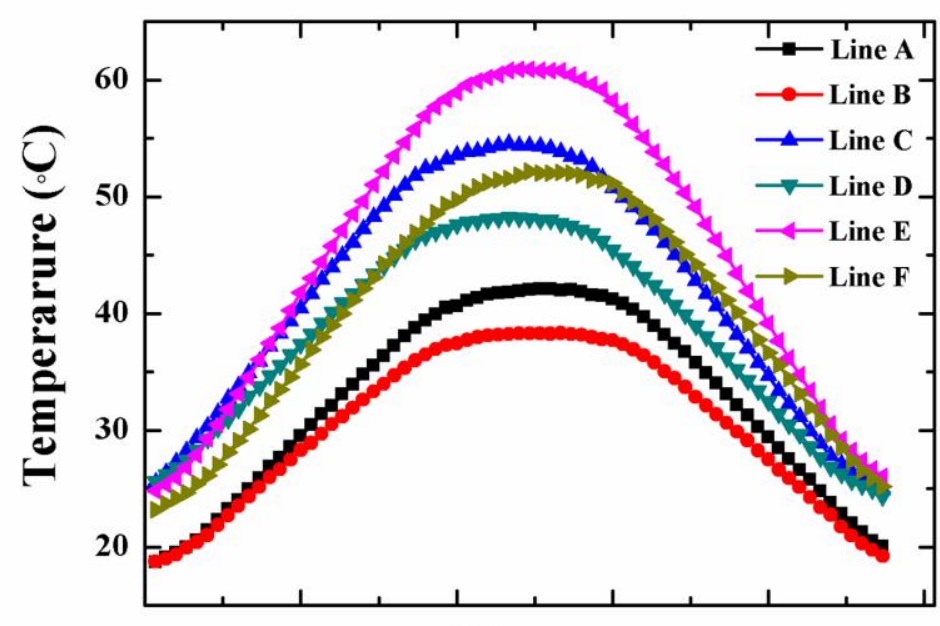

Distance 
Fig. 4. Steady-state temperature fields of testing system with homogenous composites at the heat flux of (a) $3200 \mathrm{~W} \mathrm{~m}^{-2}$, (c) $4560 \mathrm{~W} \mathrm{~m}^{-2}$ and (e) $5840 \mathrm{~W} \mathrm{~m}^{-2}$; Steady-state temperature fields of testing system with the locally reinforced composites at the heat flux of (b) $3200 \mathrm{~W} \mathrm{~m}^{-2}$, (d) $4560 \mathrm{~W} \mathrm{~m}^{-2}$ and (f) $5840 \mathrm{~W} \mathrm{~m}^{-2}$. (g) Temperature variations along the lines through the hot spots.

We further conducted the comparative experiments at the heat flux of 4560 and $5840 \mathrm{~W} \mathrm{~m}^{-2}$. Figs. 4(c)-4(f) show the temperature contours of those experiments. Similar results can be found. At the heat flux of $4560 \mathrm{~W} \mathrm{~m}^{-2}$, the reduction of $T_{\text {ave }}$ and $T_{\max }\left(\Delta T_{\text {ave }}\right.$ and $\left.\Delta T_{\max }\right)$ are $5.9{ }^{\circ} \mathrm{C}$ and $6.3{ }^{\circ} \mathrm{C}$, respectively. Furthermore, $\Delta T_{\text {ave }}$ and $\Delta T_{\max }$ increase to $7.7^{\circ} \mathrm{C}$ and $8.7^{\circ} \mathrm{C}$ when the heat flux is set to be $5840 \mathrm{~W} \mathrm{~m}^{-2}$. Fig. $4(\mathrm{~g})$ also compares the temperature variations along the lines through the hot spots. Lower temperature gradients are also found at the heater assembled with the locally reinforced composites.

\subsection{Finite element analysis of composites loaded with local heat source}

To analyze the experimental results, we simulated the heat propagation in the testing system using the finite-element-analysis (FEA) (for details, see the Appendix A). Figs. 5(a)-5(b) show the representative heat source-composite layer-heat sink models. The composite layer is divided into three bodies and the width of body $1\left(d_{1}\right)$ is equal to that of linear stripe. There are two cases to be simulated according to the comparative experiments. The first case is that particles homogeneously disperse in matrix at the composite layer (Fig. 5(a)). This is corresponding to the homogeneous case of experiments. For this case, body 1, 2 and 3 have the same particle volume faction (5\%). The second is heterogeneous case (Fig. 5(b)). Body 1 is assumed to homogeneously contain all the platelets. Correspondingly, bodies 2 and 3 are regarded as the pure matrix without any reinforcing elements. Since the width of composite is $l(26 \mathrm{~mm})$, body 1 has the volume fraction of $5 \% \times l / d_{1}(19 \%)$. Thermal conductivities $(k)$ of those bodies are the key parameters for the simulation. In this work, we used a modified effective medium approximation (EMA) [30] to predict $k$ of the bodies according to their volume fraction (for details, see the Appendix B). With EMA, $k$ of homogeneous composites is estimated to be $0.29 \mathrm{~W} \mathrm{~m}^{-1} \mathrm{~K}^{-1}$; for the heterogeneous case, $k$ of body 1 is calculated to be $0.70 \mathrm{~W} \mathrm{~m}^{-1}$ $\mathrm{K}^{-1}$, while $k$ of body 2 and 3 are equal to that of silicone gel $\left(0.16 \mathrm{~W} \mathrm{~m}^{-1} \mathrm{~K}^{-1}\right)$. In this simulation, the heat flux of heater $\left(q_{0}\right)$ is fixed to be $5840 \mathrm{~W} \mathrm{~m}^{-2}$. 
a

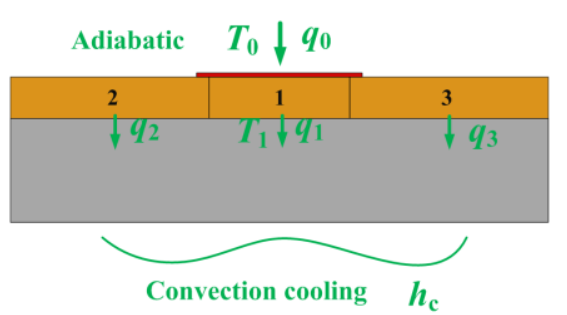

C

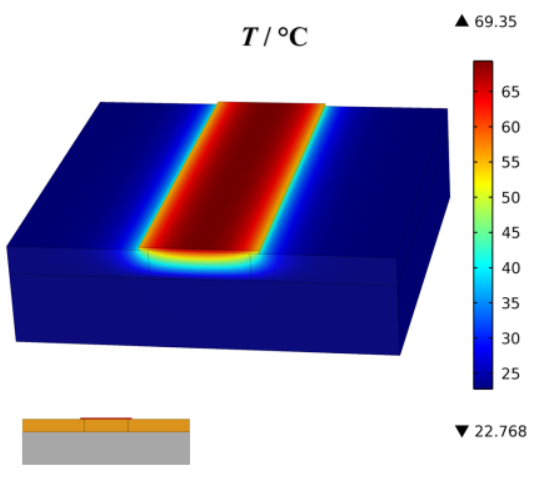

e

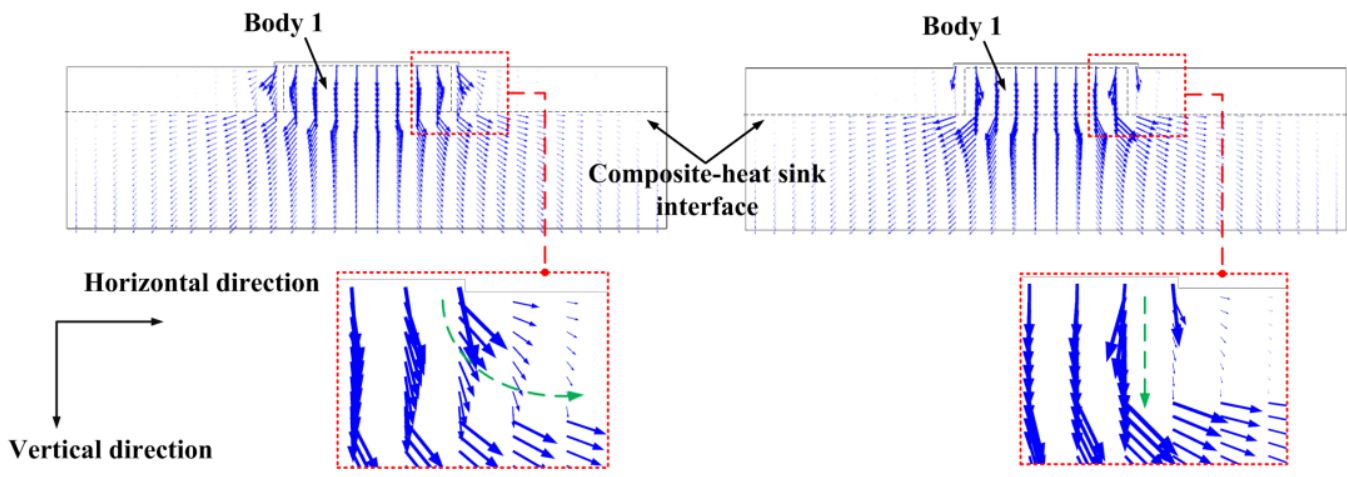

Fig. 5. Representative heat source-composite layer-heat sink models for simulation: (a) homogeneous case and (b) heterogeneous case; (c) Simulated steady state temperature field of homogeneous case, $T_{\text {ave }}$ and $T_{\max }$ are equal to $65.5^{\circ} \mathrm{C}$ and $69.4^{\circ} \mathrm{C}$, respectively; (d) Simulated steady state temperature field of heterogeneous case, $T_{\text {ave }}$ and $T_{\max }$ are equal to $51.0{ }^{\circ} \mathrm{C}$ and $53.0{ }^{\circ} \mathrm{C}$, respectively. (e and f ) Simulated cut plane vectors of heat flux inside the whole material domain of the two cases, respectively.

Figs. 5(c)-5(d) show the simulated temperature fields of the two cases, respectively. It is found that heat source attached on the locally reinforced composites exhibits lower $T_{\text {ave }}$ and $T_{\max }$. The results accords with the experimental results. With the simulated temperature fields, $\Delta T_{\text {ave }}$ and $\Delta T_{\max }$ are calculated to be $14.5^{\circ} \mathrm{C}$ and $16.4^{\circ} \mathrm{C}$, respectively. It is interesting to note that the values of $\Delta T_{\text {ave }}$ and 
$\Delta T_{\max }$ estimated by simulation are larger than those derived from experimental results. There could be two reasons lead to the overprediction of simulation. The first one is due to the assumption that at heterogeneous case, all particles are concentrated at the space of body 1. However, as shown in Fig. 2(b), the magnetic field gradient cannot attract all particles to body 1 . This assumption leads to the overprediction of filler volume fraction of body 1 and hence overprediction of thermal conductivity. The overprediction of thermal conductivity leads to overprediction of $\Delta T_{\text {ave }}$ and $\Delta T_{\max }$ in the end. Second, the boundary conditions of simulation are not the same with the experiments. For example, Heat transfer from the heater to ambient is neglected in simulation resulting in a larger $q_{0}$ compared to the experiments. And the larger $q_{0}$ will leads to the increase of $\Delta T_{\text {ave }}$ and $\Delta T_{\max }$.

Furthermore, Figs. 5(e)-5(f) present the simulated vectors of heat flux inside the whole material domain. Through the vectors, we can observe the direction of heat propagation in the composite layer. The vectors reveal that heat generated from heat source inclines to pass through the body 1 to heat sink and goes to ambient ultimately. However, the vectors near the edges of body 1 for the two cases show some difference. Figs. 5(e)-5(f) also give the partial enlarged drawings of the vectors near the edges of body 1 . It is observed that in the homogeneous composites, the heat flux at the regions tends to propagate in horizontal direction. Whereas, in the locally reinforced composites, the orientation of heat flux at the regions is still normal to the plane of composite layer. The results illustrate that the locally reinforced composites can make most of heat pass through the shortest path to reach the heat sink.

With the simulation results, we conduct a qualitative analysis to explain why heat source attached on the locally reinforced composites exhibits lower temperature. Heat flux $\left(q_{1}, q_{2}\right.$ and $\left.q_{3}\right)$ at the three bodies are calculated from the simulation results, respectively. Since heat transfer from the heater and composites to ambient are neglected in the model (see the Appendix A), heat flux of the source $q_{0}$ is the total of $q_{1}, q_{2}$ and $q_{3} . q_{1} / q_{0}$ is calculated as $71.7 \%$ and $86.5 \%$ for homogeneous and heterogeneous cases, respectively. It is found that $q_{1}$ takes the most proportion of $q_{0}$ in the two cases. Thus, we assume a 1D heat conduction of body 1 . According to Fourier's law,

$q_{1}=k_{1} \Delta T / t_{1}$

where $k_{1}$ is the thermal conductivity of body $1 ; t_{1}$ is the thickness of composite layer; neglecting the heat conduction at the thickness of heat source, $\Delta T$ is defined as temperature difference between the heat source $\left(T_{0}\right)$ and the upper surface of heat sink $\left(T_{1}\right)$ and expressed as:

$\Delta T=T_{0}-T_{1}$

Thus $T_{0}$ can be expressed as:

$T_{0}=q_{1} t_{1} / k_{1}+T_{1}$

here, $t_{1}$ is a constant; According to the simulation results, $T_{1}$ keep constant for the two cases, too. Now, $T_{0}$ is decided by the value of $q_{1} / k_{1}$. For the heterogeneous and homogeneous cases, $\left(q_{1} / q_{0}\right) / k_{1}$ is equal 
to 1.24 and 2.47 , respectively. Therefore, the lower value of $q_{1} / k_{1}$ can explain the lower $T_{0}$ of heat source attached on the locally structured composites.

\section{Conclusions}

In summary, this work shows that it is possible to fabricate the locally reinforced composites applied for efficient heat dissipation of local heat source. The local reinforcement was achieved by using the magnetically responsive hBN platelets as reinforcing elements and a magnetic field gradient to concentrate the elements below the heat source. To evaluate the thermal performance of the locally reinforced composites, we performed the comparative thermal tests on the homogeneous and locally reinforced samples. The results show that the locally reinforced composites can greatly reduce the average and maximum temperatures of heat source. Finally, we conducted a simulation study on the composites applied with local heat source. The simulation results show that the temperature of heat source is primarily dependent on the heat conduction below the heat source. Thus, concentrating the platelets at that place can enhance the heat conduction and hence decrease the temperature of heat source.

\section{Acknowledgment}

This work was supported partly by National Science Foundation of China (51376070, 51576078), and partly by 973 Project of The Ministry of Science and Technology of China (2011CB013105). The authors would like to thank Mr. Leng Zongyuan from Ferrotec for providing iron oxide nanoparticles.

\section{Appendix}

\section{A. Finite element analysis (FEA) of composites loaded with local heat source}

FEA has been used to study the heat diffusion in the heat source-composite layer-heat sink structure. Based on the experiments, we constructed a 3D stationary heat transfer model with three assumptions:

(1) Heat conduction at the aluminium surrounding the composites material is neglected;

(2) The upside of heater and composite and the sides of composites and aluminium block are kept adiabatic;

(3) Heat at the bottom of heat sink is dissipated to environment through the convection with an equivalent heat transfer coefficient $h_{\mathrm{c}}$.

According to the assumptions, Figs. 5(a)-(b) show the model geometry and boundary conditions. Tables A. 1 and A. 2 provide the parameters of geometry and material properties. Heat flux $q_{0}(5840 \mathrm{~W}$ $\mathrm{m}^{-2}$ ) is applied on the upper surface of the heater. The environmental temperature is set to be $15{ }^{\circ} \mathrm{C} . h_{\mathrm{c}}$ 
is evaluated to be $230 \mathrm{~W} \mathrm{~m}^{-2} \mathrm{~K}^{-1}$ by inverse analysis with the help of the experiments results. In fact, this parameter has little impact on the simulation results of $\Delta T_{\text {ave }}$ and $\Delta T_{\max }$. To prove it, we keep trying the simulations through changing $h_{\mathrm{c}}$ around $230 \mathrm{~W} \mathrm{~m}^{-2} \mathrm{~K}^{-1}$, the results of $\Delta T_{\text {ave }}$ and $\Delta T_{\max }$ present little variance. Heat diffusion was simulated by numerically solving the Fourier's equation with the given boundary conditions.

Table A. 1 Structure dimensions for FEA

\begin{tabular}{cc}
\hline Parameters & Values/mm \\
\hline$l$ & 26 \\
$w$ & 8 \\
$t_{\mathrm{s}}$ & 0.2 \\
$t_{\mathrm{c}}$ & 2 \\
$t_{\mathrm{h}}$ & 5 \\
$d_{1}$ & 6.8 \\
$d_{2}$ & 9.6 \\
$d_{3}$ & 9.6 \\
\hline
\end{tabular}

Table A. 2 Material properties for FEA

\begin{tabular}{cccc}
\hline & $\begin{array}{c}\text { Thermal conductivity/W } \\
\mathrm{m}^{-1} \mathrm{~K}^{-1}\end{array}$ & $\begin{array}{c}\text { Density/ kg } \\
\mathrm{m}^{-3}\end{array}$ & $\begin{array}{c}\text { Heat capacity/ J } \\
\mathrm{kg}^{-1} \mathrm{~K}^{-1}\end{array}$ \\
\hline Heater (Polyimide) & 0.15 & 1300 & 1100 \\
Silicone gel & 0.16 & 1140 & 1428 \\
5 vol \% Composites & 0.29 & 1197 & 1361 \\
19 vol \% Composites & 0.70 & 1355 & 1205 \\
Heat sink & 156 & - & - \\
(Aluminium-6061 T4) & & & \\
\hline
\end{tabular}

\section{B. Efective medium approximation for prediction of thermal conductivity of composite}

B.1 General framework

According to the effective medium approximation (EMA) theory [30], the effective thermal conductivity of the isotropic composites can be given by

$$
k=k_{m} \frac{3+f\left[2 \beta_{11}\left(1-L_{11}\right)+\beta_{33}\left(1-L_{33}\right)\right]}{3-f\left[2 \beta_{11} L_{11}+\beta_{33} L_{33}\right]}
$$

With 


$$
\begin{aligned}
& \beta_{\mathrm{ii}}=\frac{k_{\mathrm{ii}}^{c}-k_{m}}{k_{m}+L_{\mathrm{ii}}\left(k_{\mathrm{ii}}^{c}-k_{m}\right)} \\
& k_{\mathrm{ii}}^{c}=k_{p} /\left(1+\gamma L_{\mathrm{ii}} k_{p} / k_{m}\right)
\end{aligned}
$$

where $k_{m}$ and $k_{p}$ are the thermal conductivities of the matrix and fillers, respectively; $f$ is filler volume fraction; 11 and 33 represent in-plane and through-plane direction, respectively; $L_{\mathrm{ii}}$ is the geometrical factor dependent on the filler shape and given by:

$$
\begin{aligned}
& L_{11}=\frac{\alpha^{2}}{2\left(\alpha^{2}-1\right)}+\frac{\alpha}{2\left(1-\alpha^{2}\right)^{3 / 2}} \cos ^{-1} \alpha \\
& L_{33}=1-2 L_{11} \\
& \alpha=t / D
\end{aligned}
$$

where $\alpha$ is the inverse of filler aspect ratio, $t$ and $D$ are the thickness and diameter of filler, respectively. For the platelets, $\gamma$ can be given by:

$$
\gamma=(1+2 \alpha) R_{b} k_{m} / t
$$

where $R_{b}$ is thermal boundary resistance.

B. 2 Prediction of body 1 thermal conductivity in heterogeneous case

For prediction of body 1 thermal conductivity, the theoretical in-plane thermal conductivity of hBN [26-27] and the measured thermal conductivity of neat silicone gel [25] are input as known parameters:

$k_{p}=600 \mathrm{~W} \mathrm{~m}^{-1} \mathrm{~K}^{-1}, k_{m}=0.16 \mathrm{~W} \mathrm{~m}^{-1} \mathrm{~K}^{-1}$,

$D$ of hBN platelets is $5 \mu \mathrm{m}$, which is obtained from the material vendor; $t$ is approximately equal to $250 \mathrm{~nm}$; $f$ of body 1 is equal to $19 \%$. In addition, $R_{b}$ has been extracted to be $300 \times 10^{-9} \mathrm{~m}^{2} \mathrm{~W} \mathrm{~K}^{-1}$ according to Ref. [27]. With these known parameters, $k$ of body 1 is calculated using Eq. (B.1) and equal to $0.70 \mathrm{~W} \mathrm{~m}^{-1} \mathrm{~K}^{-1}$.

B.3 Prediction of homogeneous composites thermal conductivity

$k_{p}, k_{m}, t, D$ and $R_{b}$ of homogeneous composites are the same with those of body 1 . But $f$ of homogeneous composites is $5 \%$. Thus, $k$ of homogeneous composites can be also calculated using Eq. (B.1) and equal to $0.29 \mathrm{~W} \mathrm{~m}^{-1} \mathrm{~K}^{-1}$.

\section{References}

[1] Nakayama, W., 2013, "Study on Heat Conduction in a Simulated Multicore Processor Chip-Part i: Analytical Modeling," ASME J. Electron. Packag., 135(2), p. 021002. 
[2] Nakayama, W., 2013, " Study on Heat Conduction in a Simulated Multicore Processor Chip-Part ii: Case Studies, " ASME J. Electron. Packag., 135(2), p.021003.

[3] Kim, Y. J., Joshi, Y. K., Fedorov, A. G., Lee, Y. J., and Lim, S. K., 2010, "Thermal Characterization of Interlayer Microfluidic Cooling of Three-Dimensional Integrated Circuits With Nonuniform Heat Flux," ASME J. Heat Transfer, 132(4), p. 041009.

[4] Bar-Cohen, A., and Wang, P., 2012, "Thermal Management of On-Chip Hot Spot," ASME J. Heat Transfer, 134(5), p. 051017.

[5] Yi, P., Awang, R. A., Rowe, W. S., Kalantar-zadeh, K., and Khoshmanesh, K., 2014, "PDMS Nanocomposites for Heat Transfer Enhancement in Microfluidic Platforms," Lab Chip, 14(17), pp. 3419-3426.

[6] Yan, Z., Liu, G. X., Khan, J. M., and Balandin, A. A., 2012, "Graphene Quilts for Thermal Management of High-Power GaN Transistors," Nat. Commun., 3, p. 827.

[7] Kong, Q. Q., Liu, Z., Gao, J. G., Chen, C. M., Zhang, Q., Zhou, G. M., Tao, Z. C., Zhang, X. H., Wang, M. Z., and Li, F., 2014, "Hierarchical Graphene-Carbon Fiber Composite Paper as a Flexible Lateral Heat Spreader," Adv. Funct. Mater., 24(27), pp. 4222-4228.

[8] Prasher, R. S., Shipley, J., Prstic, S., Koning, P., and Wang, J. L., 2003, "Thermal Resistance of Particle Laden Polymeric Thermal Interface Materials," ASME J. Heat Transfer, 125(6), pp. $1170-1177$.

[9] Prasher, R. S., 2005, "Thermal Interface Materials: Historical Perspective, Status, and Future Directions," Proc. IEEE, 94(8), pp. 1571-1586.

[10] Sun, X. M., Sun, H., Li, H. P., and Peng, H. S., 2013, "Developing Polymer Composite Materials:Carbon Nanotubes or Graphene? " Adv. Mater., 25(37), pp. 5153-5176.

[11] Zhou, Y. C., Yao, Y. G., Chen, C. Y., Moon. K, Wang, H., and Wong, C. P., 2014, "The Use of Polyimide-Modified Aluminum Nitride Fillers in AlN@PI/Epoxy Composites with Enhanced Thermal Conductivity for Electronic Encapsulation," Sci. Rep., 4, p. 4779.

[12] Chhasatia, V., Zhou, F., Sun, Y., Huang, L. W., and Wang, H., 2008, "Design Optimization of Custom Engineered Silver-Nanoparticle Thermal Interface Materials," ITHERM, 1-3, pp. 419-427.

[13] Li, Q., Steven, G. P., Querin, O. M., and Xie, Y. M., 1999, "Shape and Topology Design for Heat Conduction by Evolutionary Structural Optimization," Int. J. Heat Mass Transfer, 42(17), 3361-3371.

[14] Xia, Z. Z., Cheng, X. G., Li, Z. X., and Guo, Z. Y., 2004, "Bionic Optimization of Heat Transport Paths for Heat Conduction Problems," J. Enhanc. Heat Transf., 11(2), pp. 119-131.

[15] Bruns. T. E., 2007, "Topology Optimization of Convection-Dominated, Steady-State Heat Transfer Problems," Int. J. Heat Mass Transfer, 50(15-16), pp. 2859-2873.

[16] Stauffer, D., and Aharony, A., 1992, Introduction to percolation theory, Taylor \& Francis, Washington, DC.

[17] Devpura, A., Phelan, P. E., and Prasher, R. S., 2000, "Percolation Theory Applied to the Analysis 
of Thermal Interface Materials in Flip-Chip Technology," ITHERM, 1, pp. 21-28.

[18] Delhaye, N., Poitou, A., and Chaouche, M., 2000, "Squeeze Flow of Highly Concentrated Suspensions of Spheres," J. Non-Newton. Fluid, 94(1), pp. 67-74.

[19] Chaari, F., Racineux, G., Poitou, A., and Chaouche, M., 2003, "Rheological Behavior of Sewage Sludge and Strain-Induced Dewatering," Rheol. Acta, 42(3), 273-279.

[20] Rae, D. F., Borgesen, P., and Cotts, E. J., 2011, "The Effect of Filler-Network Heterogeneity on Thermal Resistance of Polymeric Thermal Bondlines," JOM, 63(10), 78-84.

[21] Fujihara, T., Cho, H. B., Kanno, M., Nakayama, T., Suzuki, T., Jiang, W. H., Suematsu, H., and Niihara, K., 2014, "Three-Dimensional Structural Control and Analysis of Hexagonal Boron Nitride Nanosheets Assembly in Nanocomposite Films Induced by Electric Field Concentration," Jpn. J. Appl. Phys., 53(2), p. 02BD12.

[22] Erb, R. M., Son, H. S., Samanta, B., Rotello, V. M., and Yellen, B. B., 2009, "Magnetic Assembly of Colloidal Superstructures with Multipole Symmetry," Nature, 457(7232), 999-1002.

[23] Erb, R. M., Libanori, R., Rothfuchs, N., and Studart, A. R., 2012, "Composites Reinforced in Three Dimensions by Using Low Magnetic Fields," Science, 335(6065), 199-204.

[24] Erb, R. M., Cherenack, K. H., Stahel, R. E., Libanori, R., Kinkeldei, T., Munzenrieder, N., Troster, G., and Studart, A. R., 2012, "Locally Reinforced Polymer-Based Composites for Elastic Electronics," Acs Appl. Mater. Inter., 4(6), 2860-2864.

[25] Yuan, C., and Luo, X. B., 2013, "A Unit Cell Approach to Compute Thermal Conductivity of Uncured Silicone/Phosphor Composites," Int. J. Heat Mass Transfer, 56(1-2), 206-211.

[26] Lin, Z. Y., Liu, Y., Raghavan, S., Moon, K. S., Sitaraman, S. K., and Wong, C. P., 2013, "Magnetic Alignment of Hexagonal Boron Nitride Platelets in Polymer Matrix: Toward High Performance Anisotropic Polymer Composites for Electronic Encapsulation," Acs Appl. Mater. Interfaces, 5(15), 7633-7640.

[27] Yuan, C., Duan, B., Li, L., Xie, B., Huang, M. Y., and Luo, X. B., 2015, "Thermal Conductivity of Polymer-Based Composites with Magnetic Aligned Hexagonal Boron Nitride Platelets," Acs Appl. Mater. Interfaces, 7(23), 13000-13006.

[28] Choi, C., Yatsuzuka, K., and Asano, K., 2001, "Dynamic Motion of a Conductive Particle in Viscous Fluid under DC Electric Field," IEEE Trans. Ind. Appl. 37(3), 785-791.

[29] Wang, Y. M., Zheng, H., Hu, R., and Luo, X. B., 2014, "Modeling on phosphor sedimentation phenomenon during curing process of high power LED packaging," Journal of Solid State Lighting 1, 2.

[30] Nan, C. W., Birringer, R., Clarke, D. R., and Gleiter, H., 1997, "Effective Thermal Conductivity of Particulate Composites with Interfacial Thermal Resistance," J. Appl. Phys., 81(10), 6692-6699. 